\title{
Affine primitive groups and Semisymmetric graphs
}

\author{
Hua Han \\ Center for Combinatorics \\ LPMC, Nankai University \\ Tianjin 300071, P. R. China \\ hh1204@mail.nankai.edu.cn
}

\author{
Zai Ping $\mathrm{Lu}^{*}$ \\ Center for Combinatorics \\ LPMC, Nankai University \\ Tianjin 300071, P. R. China \\ lu@nankai.edu.cn
}

Submitted: Jul 14, 2012; Accepted: May 21, 2013; Published: May 31, 2013

Mathematics Subject Classifications: 05C25, 20B25

\begin{abstract}
In this paper, we investigate semisymmetric graphs which arise from affine primitive permutation groups. We give a characterization of such graphs, and then construct an infinite family of semisymmetric graphs which contains the Gray graph as the third smallest member. Then, as a consequence, we obtain a factorization of the complete bipartite graph $\mathrm{K}_{p^{s p^{t}}, p^{s p^{t}}}$ into connected semisymmetric graphs, where $p$ is an prime, $1 \leqslant t \leqslant s$ with $s \geqslant 2$ while $p=2$.
\end{abstract}

Keywords: semisymmetric graph; normal quotient; primitive permutation group

\section{Introduction}

All graphs considered in this paper are assumed to be finite and simple with non-empty edge sets.

For a graph $\Gamma$, denote by $V \Gamma, E \Gamma$ and Aut $\Gamma$ the vertex set, edge set and automorphism group, respectively. A graph $\Gamma$ is said to be vertex-transitive or edge-transitive if Aut $\Gamma$ acts transitively on $V \Gamma$ or $E \Gamma$, respectively. A regular graph is called semisymmetric if it is edge-transitive but not vertex-transitive. For a graph $\Gamma$, an arc of $\Gamma$ is an ordered pair $(\alpha, \beta)$ of two adjacency vertices. A graph $\Gamma$ is called symmetric if it has no isolated vertices and Aut $\Gamma$ acts transitively on the set of $\operatorname{arcs}$ of $\Gamma$.

The class of semisymmetric graphs was first studied by Folkman [9], who possed several open problem. Afterwards, many authors have done much work on this topic, see $[1,2,3,7,8,10,11,12,15,16,17,18,19]$ for references. In particular, lots of interesting examples of such graphs were found. For example, the Folkman graph on 20 vertices, the smallest semisymmetric graph, was constructed by Folkman [9]; the Gray graph, a

* Supported in part by the NSF of China. 
cubic graph of order 54, was first observed to be semisymmetric by Bouwer [1]. In 1985, Iofinova and Ivanov [11] classified all bi-primitive cubic semisymmetric graphs and they proved that there are only five such graphs.

In this paper, we consider the semisymmetric graphs whose automorphism group contains a subgroup inducing an affine primitive permutation group. For more information about groups of this kind, see $[5,6]$. To state our result, we need to introduce several concepts and some notation.

Let $p$ be a prime and $\mathbb{F}_{p}$ the field of order $p$. Then, for an integer $l \geqslant 1$ and an irreducible subgroup $H$ of the general linear group $\operatorname{GL}(l, p)$, all affine transformations $\tau_{h, v}$ form a primitive subgroup $X$ of $\operatorname{AGL}(l, p)$ (acting on the vectors of $\mathbb{F}_{p}^{l}$ ), where $h \in H$, $v \in \mathbb{F}_{p}^{l}$ and $\tau_{h, v}$ is defined as $\mathbb{F}_{p}^{l} \rightarrow \mathbb{F}_{p}^{l}, u \mapsto u^{h}+v$. The above group is a split extension

of a regular normal subgroup $\left\{\tau_{1, v} \mid v \in \mathbb{F}_{p}^{l}\right\}$ by the subgroup $H$. For convenience, for a subspace $V$ of $\mathbb{F}_{p}^{l}$, we set $\mathrm{T}(V)=\left\{\tau_{1, v} \mid v \in V\right\}$ and denote by $N_{H}(V)$ the subgroup of $H$ fixing $V$ set-wise. Then $X=\mathrm{T}\left(\mathbb{F}_{p}^{l}\right): H$, and $N_{H}(V)=\mathrm{N}_{H}(\mathrm{~T}(V))$, the normalizer of $\mathrm{T}(V)$ in $H$.

For a graph $\Gamma$ and a subgroup $G \leqslant \operatorname{Aut} \Gamma, \Gamma$ is said to be $G$-vertex-transitive or $G$-edge-transitive if $G$ is transitive on $V \Gamma$ or $E \Gamma$, respectively. The graph $\Gamma$ is called $G$-semisymmetric graphs if it is regular, $G$-edge-transitive but not $G$-vertex-transitive.

Let $\Gamma$ be a connected $G$-edge-transitive graph, where $G \leqslant$ Aut $\Gamma$. Assume that $G$ is not transitive on $V \Gamma$. Then $\Gamma$ is a bipartite graph with two parts, say $U$ and $W$, which are the $G$-orbits on $V \Gamma$. Denote respectively by $G^{U}$ and $G^{W}$ the permutation groups induced by $G$ on $U$ and on $W$. Our main result deals with the case where one of $G^{U}$ and $G^{W}$ is an affine primitive permutation group.

Theorem 1.1. Let $X=\mathrm{T}\left(\mathbb{F}_{p}^{l}\right): H$, where $p$ is a prime, $l \geqslant 1$ and $H$ is an irreducible subgroup of the general linear group $\mathrm{GL}(l, p)$. Then the following two statements are equivalent.

(1) $\mathbb{F}_{p}^{l}$ has an $s$-dimensional subspace $V$ for some integer $1 \leqslant s<l$ such that $\mid H$ : $\mathrm{N}_{H}(V) \mid=p^{t}$ for some integer $1 \leqslant t \leqslant s$.

(2) There exists a semisymmetric graph $\Gamma$ with bipartition $V \Gamma=U \cup W$ with one of $G^{U}$ and $G^{W}$ is permutation isomorphic to $X$ for some edge-transitive subgroup $G$ of Aut $\Gamma$.

Remark. Theorem 1.1 suggests the following interesting problem.

Problem 1. Characterize irreducible subgroups of the general linear group $\operatorname{GL}(l, p)$ satisfying Theorem 1.1 (1).

It is well-known that there are no semisymmetric graph of orders $16,2 p$ and $2 p^{2}$, see [9]. Thus, for Theorem $1.1(1)$, we have $l \geqslant 3$ and $(p, l) \neq(2,3)$.

\section{Proof of Theorem 1.1}

Assume that $\Gamma$ is a $G$-edge-transitive but not $G$-vertex-transitive graph, where $G \leqslant$ Aut $\Gamma$. Then $\Gamma$ is a bipartite graph with two parts, say $U$ and $W$, which are the $G$-orbits on $V \Gamma$. 
It follows that $\Gamma$ is semiregular, that is, the vertices in a same bipartition subset have the same valency. For a given vertex $\alpha \in V \Gamma$, the stabilizer acts transitively on $\Gamma(\alpha)$. Thus, if $\Gamma$ is vertex-transitive then it must be symmetric. Take $\beta \in \Gamma(\alpha)$. Then each vertex of $\Gamma$ can be written as $\alpha^{g}$ or $\beta^{g}$ for some $g \in G$. Then, for two arbitrary vertices $\alpha^{g}$ and $\beta^{h}$, they are adjacent in $\Gamma$ if and only if $\alpha$ and $\beta^{h g^{-1}}$ are adjacent, i.e., $h g^{-1} \in G_{\beta} G_{\alpha}$. Moreover, it is well-known and easily shown that $\Gamma$ is connected if and only if $\left\langle G_{\alpha}, G_{\beta}\right\rangle=G$.

Let $\Gamma$ be a $G$-semisymmetric graph with two bipartition subsets $U$ and $W$. Suppose that $G$ has a subgroup $R$ which is regular on both $U$ and $W$. Take an edge $\{\alpha, \beta\} \in E \Gamma$. Then each vertex in $U$ ( $W$, resp.) can be written uniquely as $\alpha^{x}$ ( $\beta^{x}$, resp.) for some $x \in R$. Set $S=\left\{s \in R \mid \beta^{s} \in \Gamma(\alpha)\right\}$. Then $\alpha^{x}$ and $\beta^{y}$ are adjacent if and only if $y x^{-1} \in S$. If $R$ is abelian, then it is easily shown that $\alpha^{x} \mapsto \beta^{x^{-1}}, \beta^{x} \mapsto \alpha^{x^{-1}}, \forall x \in R$ is an automorphism of $\Gamma$, which leads to the vertex-transitivity of $\Gamma$, refer to $[8,14]$.

Lemma 2.1. Let $\Gamma$ be a $G$-semisymmetric graph. Assume that $G$ has an abelian subgroup which is regular on both parts of $\Gamma$. Then $\Gamma$ is symmetric.

Let $\Gamma$ be a $G$-semisymmetric graph. Suppose that $G$ has a normal subgroup $N$ which acts intransitively on at least one of the bipartition subsets of $\Gamma$. Then we define the quotient graph $\Gamma_{N}$ to have vertices the $N$-orbits on $V \Gamma$, and two $N$-orbits $\Delta$ and $\Delta^{\prime}$ are adjacent in $\Gamma_{N}$ if and only if some $\alpha \in \Delta$ and some $\beta \in \Delta^{\prime}$ are adjacent in $\Gamma$. It is easy to see that the quotient $\Gamma_{N}$ is a regular graph if and only if all $N$-orbits have the same length.

Let $\Gamma$ be a finite connected $G$-semisymmetric graph with $G \leqslant$ Aut $\Gamma$. Take an edge $\{\alpha, \beta\} \in E \Gamma$ and let $U=\alpha^{G}$ and $W=\beta^{G}$ be the two $G$-orbits on $V \Gamma$. Assume that $G$ is unfaithful on $U$, and let $K$ be the kernel of $G$ acting on $U$. Then $K$ is faithful on $W$, and each $K$-orbits on $W$ contains at least two vertices. It follows that there are two distinct vertices in $W$ which have the same neighborhood in $\Gamma$. Thus, as observed in [8], if any two distinct vertices in $U$ have different neighborhoods in the quotient $\Gamma_{K}$ then $\Gamma$ is semisymmetric and can be reconstructed from $\Gamma_{K}$ as follows.

Construction 2.2. Let $\Sigma$ be a bipartite graph with two bipartition subset $U$ and $\bar{W}$ such that $m|\bar{W}|=|U|$ for integer $m>1$. Define a bipartite graph $\Sigma^{1, m}$ with vertex set $U \cup\left(\bar{W} \times \mathbb{Z}_{m}\right)$ such that $\alpha$ and $(\beta, i)$ are adjacent if and only if $\{\alpha, \beta\} \in E \Gamma$. For convenience, we set $\Sigma^{1,1}=\Sigma$.

For a group $X$, the socle of $X$, denoted by $\operatorname{soc}(X)$, is generated by all minimal normal subgroups of $X$. A permutation group is called quasiprimitive if each of its minimal normal subgroups is transitive.

Lemma 2.3. Let $\Gamma$ be a finite connected $G$-semisymmetric graph with $G \leqslant$ Aut $\Gamma$. Take an edge $\{\alpha, \beta\} \in E \Gamma$ and set $U=\alpha^{G}$ and $W=\beta^{G}$. Assume that $G^{U}$ is quasiprimitive. Then either $G$ is faithful on both $U$ and $W$, or one of the following statements hold.

(1) $\Gamma$ is isomorphic to the complete bipartite graph $\mathrm{K}_{|U|,|U|}$; 
(2) $G$ is faithful on $W$ but not faithful on $U, G^{U} \cong G^{\bar{W}}$, and $\Gamma$ is semisymmetric if further $G^{U}$ is primitive, where $K$ is the kernel of $G$ on $U$ and $\bar{W}$ is the set of $K$-orbits on $W$.

Proof. To prove this lemma, we assume next that $G$ is unfaithful on at least one of $U$ and $W$ and that $\Gamma \neq \mathrm{K}_{|U|,|U|}$.

Since $G \leqslant$ Aut $\Gamma$, the kernel of $G$ on one bipartition subset must be faithful on the other one. Then the above assumption implies that $G$ is faithful on $W$. Thus $G$ is unfaithful on $U$. Let $K$ and $\bar{W}$ be as in the lemma. Then $G^{U} \cong G / K$ and $G$ induces a subgroup $\bar{G} \cong G / K$ of Aut $\Gamma_{K}$. Suppose that $\bar{G}$ is unfaithful on $\bar{W}$. Then it follows that $\Gamma_{K} \cong \mathrm{K}_{|U|,|\bar{W}|}$, and so $\Gamma \cong \mathrm{K}_{|U|,|U|}$ by noting that $\beta^{K} \subseteq \Gamma(\alpha)$ if $\beta \in \Gamma(\alpha)$, a contradiction. Thus $\bar{G}$ is faithful on $\bar{W}$, so $K$ is the kernel of $G$ acting on $\bar{W}$, and hence $G^{U} \cong G / K \cong G^{\bar{W}}$.

Note that each $K$-orbit on $W$ has size at least 2 , and that two vertices in a same $K$-orbit have the same neighborhood in $\Gamma$. Assume that $G^{U}$ is primitive. Then, since $\Gamma \neq \mathrm{K}_{|U|,|U|}$, any two distinct vertices in $U$ have different neighborhood. Thus there is no $x \in$ Aut $\Gamma$ with $U^{x}=W$, so $\Gamma$ must be semisymmetric.

Corollary 2.4. Let $G$ a finite group which acts faithfully and transitively on both nonempty sets $U$ and $\bar{W}$ with $|U|=m|\bar{W}|$ for an integer $m>1$. For $\alpha \in U$ and a $G_{\alpha^{-}}$orbit $\Theta$ on $\bar{W}$, define a bipartite graph $\Sigma$ on $U \cup \bar{W}$ such that $\alpha^{g} \in U$ and $\bar{\beta} \in \bar{W}$ are adjacent if and only if $\bar{\beta} \in \Theta^{g}$. If $G^{U}$ is primitive, then $\Sigma^{1, m}$ is a semisymmetric graph unless $\Theta=\bar{W}$.

Proof. Assume that $G^{U}$ is primitive and $\Theta \neq \bar{W}$. By Lemma 2.3, it suffices to show that Aut $\Sigma^{1, m}$ has an edge-transitive subgroup which fixes $U$ and induces a permutation group on $U$ permutation isomorphic to $G^{U}$. Let $Y=G \times \mathbb{Z}_{m}$. Define an action of $Y$ on $V \Sigma^{1, m}$ as follows:

$$
\left(\alpha^{g}\right)^{(x, i)}=\alpha^{g x},(\bar{\beta}, j)^{(x, i)}=\left(\bar{\beta}^{x}, i+j\right), \forall g, x \in G, i, j \in \mathbb{Z}_{m}, \bar{\beta} \in \bar{W} .
$$

Then, under the above action, $Y$ is a subgroup of Aut $\Sigma^{1, m}$ as desired.

Remark. A graph is called edge-primitive if its automorphism group is primitive on its edge set. Using Corollary 2.4, we can construct examples of semisymmetric graphs from an edge-primitive graph of even valency, such as the complete graph $\mathrm{K}_{2 l+1}$, the Perkel graph and etc., by taking $U, \bar{W}$ and $\Theta$ respectively the edge set, vertex set and an orbit on $\bar{W}$ of some edge-stabilizer of the edge-primitive graph.

Here we pose the next interesting problem.

Problem 2. Characterize or classify the primitive subgroups of $\mathrm{S}_{n}$ which have transitive permutation representations of degree properly dividing $n$.

Lemma 2.5. Let $\Gamma$ be a connected $G$-semisymmetric graph with $G \leqslant$ Aut $\Gamma$. Take an edge $\{\alpha, \beta\} \in E \Gamma$ and set $U=\alpha^{G}$ and $W=\beta^{G}$. Assume that $G$ is faithful on both $U$ and $W$. Assume that $G^{U}$ is an affine primitive permutation group and $\Gamma$ is not a complete bipartite graph. Then either $G$ is primitive on $W$, or $\Gamma$ is semisymmetric. 
Proof. Set $N=\operatorname{soc}(G)$. Then $N \cong \mathbb{Z}_{p}^{l}$ for a prime $p$ and integer $l \geqslant 1$. It is easily shown that $G$ is primitive on $W$ if and only if $N$ is transitive on $W$. To finish the proof, in the following, we prove that $\Gamma$ is semisymmetric if $N$ is intransitive on $W$.

Suppose that $N$ is intransitive on $W$ and $\Gamma$ is symmetric. Then $N_{\gamma} \neq 1$ for any $\gamma \in W$. Let $X$ be the set-wise stabilizer of $U$ in Aut $\Gamma$. Then $G \leqslant X$ and $\mid$ Aut $\Gamma: X \mid=2$. Note that $X^{U}$ is a primitive permutation group. By Lemma 2.3, since $\Gamma$ is not a complete bipartite graph, we may assume that $X$ is faithful on both $U$ and $W$. Let $M=\operatorname{soc}(X)$. Take $x \in$ Aut $\Gamma$ with $\alpha^{x}=\beta$ and $\beta^{x}=\alpha$. Then $U^{x}=W$ and $W^{x}=U$. Note that $X$ is a primitive permutation group (on $U$ ) of degree $p^{l}$. Then $M$ is the unique minimal normal subgroup of $X$ by the O'Nan-Scott Theorem, refer to [4, Theorem 4.1A]. Thus $M^{x}=M$, it follows that $M$ is transitive on both $U$ and $W$. If $X$ is of affine type then $M=N$ by [20, Proposition 5.1], so $N$ is transitive on $U$, a contradiction. Then, by [13] and [20, Proposition 5.1], $N<M$ and $M$ is listed as follows:

(i) $M \cong T^{l}$, where $(p, T)$ is one of $(11, \operatorname{PSL}(2,11)),\left(11, \mathrm{M}_{11}\right),\left(23, \mathrm{M}_{23}\right)$ and $\left(\frac{q^{d}-1}{q-1}, \operatorname{PSL}(d, q)\right)$; or

(ii) $M=T_{1} \times \cdots \times T_{t}$ and $N=\left(N \cap T_{1}\right) \times \cdots \times\left(N \cap T_{t}\right)$, where $T_{i}=\mathrm{A}_{p^{s}}$ and $N \cap T_{i} \cong \mathbb{Z}_{p}^{s}$ for $1 \leqslant i \leqslant t, p^{s} \geqslant 5$ and $s t=l$.

For (i) and (ii) with $s=1$, the stabilizer $M_{\alpha}$ has order coprime to $p$, and so does for $M_{\alpha}^{x}=M_{\beta}$, hence $N \cap M_{\beta}=1$, which contradicts that $N_{\beta} \neq 1$. Thus (ii) occurs and $s \geqslant 2$, so $p^{s}>5$. It is easily shown that $\left(T_{i}\right)_{\alpha} \cong \mathrm{A}_{p^{s}-1}$ and $M_{\alpha}=\left(T_{1}\right)_{\alpha} \times \cdots \times\left(T_{t}\right)_{\alpha}$. Then $M_{\beta}=$ $M_{\alpha}^{x}=\left(\left(T_{1}\right)_{\alpha} \times \cdots \times\left(T_{t}\right)_{\alpha}\right)^{x}=\left(\left(T_{1} \cap M_{\alpha}\right) \times \cdots \times\left(T_{t} \cap M_{\alpha}\right)\right)^{x}=\left(T_{1}\right)_{\beta} \times \cdots \times\left(T_{t}\right)_{\beta}$. It implies that $\left\{\left(T_{1}\right)_{\alpha}, \cdots,\left(T_{t}\right)_{\alpha}\right\}^{x}=\left\{\left(T_{1}\right)_{\beta}, \cdots,\left(T_{t}\right)_{\beta}\right\}$ as all $\left(T_{i}\right)_{\alpha}$ are simple and nonabelian. In particular, $\left(T_{i}\right)_{\beta} \cong \mathrm{A}_{p^{s}-1}$, so $\left(T_{i}\right)_{\alpha}$ and $\left(T_{i}\right)_{\beta}$ are conjugate in $T_{i}$ for $1 \leqslant i \leqslant t$. Thus $M_{\alpha}$ and $M_{\beta}$ are conjugate in $M$. Since $M$ is transitive on $W$, there is $\gamma \in W$ with $M_{\alpha}=M_{\gamma}$. Then $N_{\gamma}=N \cap M_{\gamma}=N \cap M_{\alpha}=1$ as $N$ is regular on $U$, again a contradiction. This completes the proof.

Remark It is well-known that there are no symmetric graphs of order $2 p$ and $2 p^{2}$. Thus, for Lemma 2.5, if $N$ is intransitive on $W$ then $N \cong \mathbb{Z}_{p}^{l}$ for $l \geqslant 3$, which also follows from checking the irreducible subgroups of $\mathrm{GL}(2, p)$.

Proof of Theorem 1.1. Let $\Gamma$ be a semisymmetric graph with bipartition $V \Gamma=U \cup W$ satisfying Theorem 1.1 (2). Then $\Gamma$ must be connected. Without loss of generality, we assume that $G^{U}$ is permutation isomorphic to $X$. By Lemma 2.3, $G$ is faithful on $W$. Let $K$ be the kernel of $G$ acting on $U$ and $\bar{W}$ be the set of $K$-orbits on $W$, while $K=1$ and $\bar{W}=W$ if $G$ is faithful on $U$. Then, by Lemmas 2.3 and $2.5, \Gamma_{K}$ is edge-transitive but not vertex-transitive. Let $\bar{G}$ be the subgroup of Aut $\Gamma_{K}$ induced by $G$. Then $\bar{G} \cong G / K$, and $\bar{G}^{U}$ is permutation isomorphic to $X$.

Set $N=\operatorname{soc}(\bar{G})$. Suppose that $N$ is transitive on $\bar{W}$. Then, since $N$ is faithful on $\bar{W}$, we have $|\bar{W}|=|N|=|U|$. Thus $K=1$ and $\Gamma=\Gamma_{K}, \Gamma$ is symmetric by Lemma 2.1, a contradiction. Then $N$ is intransitive on $\bar{W}$. Take an edge $\{\alpha, \bar{\beta}\}$ of $\Gamma_{K}$ with $\alpha \in U$ and $\bar{\beta} \in \bar{W}$. Then each $N$-orbit on $\bar{W}$ has size $\left|N: N_{\bar{\beta}}\right| \neq 1$, and $N$ has exactly $\frac{|\bar{W}|}{\left|N: N_{\bar{\beta}}\right|}$ orbits on $\bar{W}$. Set $\left|N_{\bar{\beta}}\right|=p^{s}$ and $\frac{|\bar{W}|}{\left|N: N_{\bar{\beta}}\right|}=p^{r}$. Then $1 \leqslant r \leqslant s<l$. Since 
$\bar{G}$ is transitive on $\bar{W}$, we know that $\bar{G}_{\alpha}$ is transitive on the set of $N$-orbits. Let $B$ be an $N$-orbit containing $\bar{\beta}$. Then $\left|\bar{G}_{\alpha}:\left(\bar{G}_{\alpha}\right)_{B}\right|=p^{r}$. Since $\bar{G}_{\alpha}$ is maximal in $\bar{G}$, we have $\bar{G}_{\alpha} \nless \mathrm{N}_{\bar{G}}\left(N_{\bar{\beta}}\right)$. So $\left|\bar{G}_{\alpha}: \mathrm{N}_{\bar{G}_{\alpha}}\left(N_{\bar{\beta}}\right)\right|>1$. Consider the set-wise stabilizer $\left(\bar{G}_{\alpha}\right)_{B}$. For $g \in\left(\bar{G}_{\alpha}\right)_{B}$, noting that $N_{\bar{\beta}}$ is the kernel of $N$ acting on $B$, we have $\bar{\beta}^{g x}=\bar{\beta}^{g}$ for $x \in N_{\bar{\beta}}$, so $g x g^{-1} \in N \cap \bar{G}_{\bar{\beta}}=N_{\bar{\beta}}$. Thus $\left(\bar{G}_{\alpha}\right)_{B} \leqslant \mathrm{~N}_{\bar{G}_{\alpha}}\left(N_{\bar{\beta}}\right)$. Set $\left|\bar{G}_{\alpha}: \mathrm{N}_{\bar{G}_{\alpha}}\left(N_{\bar{\beta}}\right)\right|=p^{t}$. Then $t \geqslant 1$, and $p^{t}$ divides $\left|\bar{G}_{\alpha}:\left(\bar{G}_{\alpha}\right)_{B}\right|=p^{r}$, so $t \leqslant r \leqslant s$. Noting that $\bar{G}^{U}$ is permutation isomorphic to $X,(1)$ of Theorem 1.1 follows.

Now we assume that Theorem 1.1 (1) holds. To show (2), it suffices to construct a suitable semisymmetric graph. Set $R=\mathrm{T}(V) \mathrm{N}_{H}(V)$. Let $\bar{W}$ be the set of right cosets of $R$ in $X$, and let $U$ be the set of vectors in $\mathbb{F}_{p}^{l}$. Then $|\bar{W}|=p^{l-s+t}$. Extend $X$ to a permutation group on $U \cup \bar{W}$ such that $X^{U}=X$ and $X$ acts on $\bar{W}$ by the right multiplication on the right cosets of $R$ in $X$. It is easily shown that $X$ is faithful on both $U$ and $\bar{W}$. Let $\Theta=\{R h \mid h \in H\}$. Then $|\Theta|=\frac{|V||H|}{|R|}=\left|H: \mathrm{N}_{H}(V)\right|=p^{t}<|\bar{W}|$. Define a bipartite graph $\Sigma$ on $U \cup \bar{W}$ such that $u \in U$ and $\bar{\beta} \in \bar{W}$ are adjacent if and only if $\bar{\beta} \in \Theta^{\tau_{1, u}}$. Then $\Sigma$ is $X$-edge-transitive. Let $m=p^{s-t}$. If $m=1$ then, by Lemma 2.5, $\Sigma$ is a semisymmetric graph as desired. If $m>1$ then, by Corollary $2.4, \Sigma^{1, m}$ is a semisymmetric graph as desired. This completes the proof.

\section{Some examples}

Let $X=\mathrm{T}\left(\mathbb{F}_{p}^{l}\right): H$ be a primitive permutation group satisfying Theorem 1.1 (1). Then, up to isomorphism, each graph satisfying Theorem 1.1 (2) can be constructed from an $X$-edge-transitive graph which is not a complete bipartite graph and has one bipartition subset coinciding with the underlaying set of $\mathbb{F}_{p}^{l}$. Let $\Sigma$ be such an $X$-edge-transitive graph with two bipartition subsets $U=\mathbb{F}_{p}^{l}$ and $W$. Then, for each $\beta \in W$, the stabilizer $X_{\beta}$ normalizes $\left(\mathrm{T}\left(\mathbb{F}_{p}^{l}\right)\right)_{\beta}=\mathrm{T}(V)$, where $V$ is an $s$-dimensional subspace of $U$ and $X_{\beta} \cap \mathrm{T}\left(\mathbb{F}_{p}^{l}\right)=$ $\mathrm{T}(V)$. Thus $X_{\beta} \leqslant \mathrm{N}_{X}(\mathrm{~T}(V))=\mathrm{T}\left(\mathbb{F}_{p}^{l}\right) \mathrm{N}_{H}(V)$. Assume that $\mathrm{T}\left(\mathbb{F}_{p}^{l}\right)$ has $p^{r}$ orbits on $W$, and set $\left|H: \mathrm{N}_{H}(V)\right|=p^{t}$. Then $1 \leqslant t \leqslant r \leqslant s<l$, and $\left|X_{\beta}\right|=p^{s-r}|H|$. We next consider one extreme case.

Assume that $X_{\beta}=\mathrm{T}(V): \mathrm{N}_{H}(V)$. Then $r=t$, and $W$ may be identified with $\cup_{h \in H}\{u+$ $\left.V^{h} \mid u \in \mathbb{F}_{p}^{l}\right\}$ with the action of $X$ on $W$ as follows:

$$
\left(u+V^{h}\right)^{\tau_{1, u^{\prime}} h^{\prime}}=\left(u+u^{\prime}\right)^{h^{\prime}}+V^{h h^{\prime}} \forall u, u^{\prime} \in \mathbb{F}_{p}^{l}, h, h^{\prime} \in H .
$$

For each $u \in U=\mathbb{F}_{p}^{l}$, set $\Theta(u)=\left\{u^{h}+V^{h} \mid h \in H\right\}$. Then $\left\{\Theta(u) \mid u \in \mathbb{F}_{p}^{l}\right\}$ is the set of $H$-orbits on $W$. Moreover, $|\Theta(0)|=p^{t}<|W|=p^{l-s+t}$, and so $|\Theta(u)|<|W|$ for each $u \in U$. Thus $\Sigma$ is isomorphic to one of the graphs constructed as follows.

Construction 3.1. Let $p, l, H$ and $V$ be as in Theorem 1.1 (1). Let $U=\mathbb{F}_{p}^{l}$ and $W=\cup_{h \in H}\left\{u+V^{h} \mid u \in \mathbb{F}_{p}^{l}\right\}$. For each $u_{0} \in U$, define a bipartite graph $\Gamma\left(p, l, s, t ; H, u_{0}\right)$ on $U \cup W$ such that $u \in U$ and $u^{\prime}+V^{h^{\prime}} \in W$ are adjacent if and only if

$$
u^{\prime}+V^{h^{\prime}}=u+u_{0}^{h}+V^{h} \text { for some } h \in H,
$$


i.e.,

$$
u_{0}+\left(u-u^{\prime}\right)^{h^{-1}} \in V \text { and } h^{\prime} h^{-1} \in N_{H}(V) \text { for some } h \in H .
$$

The next result follows from Lemmas 2.3 and 2.5, Corollary 2.4 and the above argument.

Corollary 3.2. If there is a graph $\Sigma=\Gamma\left(p, l, s, t ; H, u_{0}\right)$ as in Construction 3.1, then $\Sigma^{1, p^{s-t}}$ is semisymmetric.

Now we construct an infinite family of semisymmetric graphs.

Example 3.3. Let $p$ be a prime, and let $s$ and $t$ be two integers with $1 \leqslant t \leqslant s$ such that $s \geqslant 2$ if further $p=2$. Let $l=s p^{t}$. Write $\mathbb{F}_{p}^{l}$ in a direct sum

$$
\mathbb{F}_{p}^{l}=\bigoplus_{i=1}^{p^{t}} U_{i}
$$

of $s$-dimensional subspaces. Without loss of generality, for each $i$, we take $\left\{e_{i j} \mid 1 \leqslant j \leqslant s\right\}$ as a basis of $U$, where $e_{i j}$ is the column vector with the $((i-1) s+j)$-th entry equal to 1 and the other entries equal to zero.

Let $H$ be the subgroup of $\operatorname{GL}(l, p)$ fixes the above decomposition. Then $H \cong \operatorname{GL}(s, p)$ r $\mathrm{S}_{p^{t}}$. Let $V=U_{1}$ and $\Sigma=\Gamma(p, l, s, t ; H, u)$. Set

$$
G(p, s, t ; u)=\Sigma^{1, m}, \text { where } m=p^{s-t}
$$

with $\Sigma^{1, m}=1$ while $m=1$. Then we get a family

$$
\mathcal{G}=\left\{G(p, s, t ; u) \mid 1 \leqslant t \leqslant s, p \text { is a prime, }(p, s) \neq(2,1), u \in \mathbb{F}_{p}^{s p^{t}}\right\}
$$

of semisymmetric graphs, and the following statements hold:

(i) $G(p, s, t ; 0)$ has valency $p^{s}=p^{t} p^{s-t}$;

(ii) $G(p, s, t ; u)=G\left(p, s, t ; u^{\prime}\right)$ if $u-u^{\prime} \in U_{i}$ for some $1 \leqslant i \leqslant p^{t}$;

(iii) $G\left(p, s, t ; e_{i 1}\right)=G\left(p, s, t ; e_{i^{\prime} 1}\right)$ for $i, i^{\prime} \geqslant 2$;

(iv) $G\left(p, s, t ; e_{21}\right)$ has valency $p^{s}\left(p^{s}-1\right)\left(p^{t}-1\right)$;

(v) $G\left(p, s, t ; \sum_{a=1}^{k} u_{i_{a}}\right)=G\left(p, s, t ; \sum_{a=1}^{k} e_{i_{a} 1}\right)$ for $2 \leqslant i_{1}<i_{2}<\cdots i_{k} \leqslant p^{t}$ and $u_{i} \in$ $U_{i} \backslash\{0\}$

(vi) $G\left(p, s, t ; \sum_{i=2}^{k} e_{i 1}\right)$ has valency $p^{s}\left(p^{s}-1\right)^{k-1}\left(\begin{array}{c}p^{t}-1 \\ k-1\end{array}\right)$, where $2 \leqslant k \leqslant p^{t}$.

Therefore the complete graph $K_{p^{s p^{t}}, p^{s p^{t}}}$ can be factorized into $p^{t}$ connected semisymmetric graphs. In particular, $K_{27,27}$ is the edge-disjoint union of three semisymmetric graphs of valency 3,12 and 12 , say, $G(3,1,1 ; 0), G\left(3,1,1 ; e_{21}\right)$ and $G\left(3,1,1 ; e_{21}+e_{31}\right)$.

By [3], there is a unique cubic semisymmetric graph of order 54 . Thus $G(3,1,1 ; 0)$ is in fact the Gray graph. The smallest members of $\mathcal{G}$ have order 32 , which are $G(2,2,1 ; 0)$ and $G\left(2,2,1 ; e_{21}\right)$ and have valency 4 and 12 , respectively. 
It is easily shown that, for $s \geqslant 2$, we can get the same graphs as in Example 3.3 if replace $H$ by $H \cap \operatorname{SL}(l, p)$. This is also true for $s=1$ unless $p=3$.

Example 3.4. Let $p$ be an odd prime. Write $\mathbb{F}_{p}^{p}$ in a direct sum $\mathbb{F}_{p}^{p}=\bigoplus_{i=1}^{p} U_{i}$ of 1dimensional subspaces. Assume that $e_{i} \in U_{i}$ for each $i$, where $e_{i}$ is the column vector with the $i$-th entry equal to 1 and the other entries equal to zero. Let $H$ be the subgroup of $\operatorname{SL}(p, p)$ fixes the above decomposition. Then $H \cong \mathbb{Z}_{p-1}^{p-1}: \mathrm{A}_{p}$. Let $V=U_{1}$ and

$$
S(p, p ; u)=\Gamma(p, p, 1,1 ; H, u) .
$$

Then each $S(p, p ; u)$ is semisymmetric graph, and the following statements hold:

(i) $S(p, p ; 0)=G(p, 1,1 ; 0)$ has valency $p$;

(ii) $S\left(p, p ; e_{i}\right)=G\left(p, 1,1 ; e_{i}\right)$ has valency $p(p-1)^{2}$, for $p \geqslant 5$ and $2 \leqslant i \leqslant p$;

(iii) $S\left(3,3 ; e_{2}\right)$ and $S\left(3,3 ; e_{3}\right)$ have valency 6 , and $G\left(3,1,1, e_{2}\right)$ is the edge-disjoint union of these two graphs;

(iv) $S\left(p, p ; \sum_{i=2}^{k} e_{i}\right)=G\left(p, 1,1 ; \sum_{i=2}^{k} e_{i}\right)$ has valency $p(p-1)^{k-1}\left(\begin{array}{c}p-1 \\ k-1\end{array}\right)$ for $k \geqslant 3$.

\section{References}

[1] I. Z. Bouwer. An edge but not vertex transitive cubic graphs. Canad Math. Bull., 11:533-535, 1968.

[2] I. Z. Bouwer. On edge but not vertex transitive graphs. J. Combin. Theory Ser. B, 12:32-40, 1972.

[3] M. Conder, A. Malnič, D. Marušič and P. Potočnik. A census of semisymmetric cubic graphs on up to 768 vertices. J. Algebr. Comb., 23:255-294, 2006.

[4] J. D. Dixon and B. Mortimer. Permutation Groups. Springer-Verlag New York Berlin Heidelberg, 1996.

[5] R. Lyndon. Groups and Geometry. Cambridge University Press, Cambridge, 1985.

[6] R. Artzy. Linear Geometry. Addison-Wesley, 1965.

[7] S. F. Du and D. Marušič. Biprimitive Graphs of Smallest Order. J Algebr. Comb., 9:151-156, 1999.

[8] S. F. Du and M. Y. Xu. A classification of semisymmetric graphs of order $2 p q$. Comm. Algebra, 28(6):2685-2714, 2000.

[9] J. Folkman. Regular line-symmetric graphs. J. Combin.Theory Ser. B, 3:215-232, 1967.

[10] H. Han and Z. P. Lu. Semisymmetric graphs of order $6 p^{2}$ and prime valency. Sci. China Math., 55:2579-2592, 2012. 
[11] M. E. Iofinova and A. A. Ivanov. Biprimitive cubic graphs (Russian), in: Investigation in Algebraic Theory of Combinatorial Objects, Proc. of the Seminar, Institute for System Studies, Moscow, pp.124-134, 1985.

[12] A. V. Ivanov. On edge but not vertex transitive regular graphs. Ann. Discrete Math., 34:273-286, 1987.

[13] C. H. Li. The finite primitive permutation groups containing an abelian regular subgroup, Proc. London Math. Soc., 87(3):725-747, 2003.

[14] Z. P. Lu. On the automorphism groups of bi-Cayley graphs. Beijing Daxue Xuebao $39: 1-5,2003$.

[15] Z. P. Lu, C. Q. Wang and M. Y. Xu. On Semisymmetric Cubic Graphs of Order $6 p^{2}$. Sci.China Ser.A , 47:1-17, 2004.

[16] A. Malnič, D. Marušič and C. Q. Wang. Cubic edge-transitive graphs of order $2 p^{3}$. Discrete Math., 274:187-198, 2004.

[17] D. Marušič and P. Potočnik. Semisymmetry of generalized Folkman graphs. European J. Combin., 22:333-349, 2001.

[18] B. Monson, T. Pisanski, E. Schulte and A. I. Weiss. Semisymmetric graphs from polytopes. J. Combin. Theory Ser. A, 114:421-435, 2007.

[19] C. W. Parker. Semisymmetric cubic graphs of twice odd order. European J. Combin., 28:572-591, 2007.

[20] C. E. Praeger. The inclusion problem for finite primitive permutation groups. Proc. London Math. Soc., 60(3):68-88, 1990. 